\title{
Médiévales
}

Langues, Textes, Histoire

75 | automne 2018

Traductions du Moyen Âge

\section{Y a-t-il de l'intraduisible? Quelques exemples de résistances dans les écrits scientifiques du Moyen Âge}

Are some things untranslatable? Certain cases of resistance in the scientific writings of the Middle Ages

Laurence Moulinier-Brogi

\section{OpenEdition \\ Journals}

Édition électronique

URL : https://journals.openedition.org/medievales/9283

DOI : $10.4000 /$ medievales.9283

ISSN : $1777-5892$

\section{Éditeur}

Presses universitaires de Vincennes

\section{Édition imprimée}

Date de publication : 15 octobre 2018

Pagination : 117-136

ISBN : 978-2-84292-861-2

ISSN : 0751-2708

\section{Référence électronique}

Laurence Moulinier-Brogi, « Y a-t-il de l'intraduisible? Quelques exemples de résistances dans les écrits scientifiques du Moyen Âge », Médiévales [En ligne], 75 | automne 2018, mis en ligne le 15 octobre 2019, consulté le 22 avril 2022. URL : http://journals.openedition.org/medievales/9283 ; DOI : https://doi.org/10.4000/medievales.9283 
Laurence Moulinier-Brogi

\section{Y a-t-il de l'intraduisible? \\ Quelques exemples de résistance dans les écrits scientifiques du Moyen Âge}

Le Moyen Âge nous a légué une très vaste littérature que, faute de mieux, on qualifiera de technique ou scientifique, sans être satisfait de cette appellation qui n'arrive pas à recouvrir la notion de Fachliteratur en allemand. Est-il plus satisfaisant de dire « littérature spécialisée » pour qualifier cette littérature... non « littéraire »? Quoi qu'il en soit, les textes que je souhaite envisager ici ont tous pour ambition de décrire, d'expliquer le réel, ou de transmettre un savoir à son sujet, de la plante aux astres en passant par le corps humain, et plusieurs spécificités nous invitent à leur faire une place dans ce dossier sur la traduction : peu ou mal édités, ces textes soulèvent des problèmes terminologiques qui furent parfois et restent souvent autant d'écueils à leur compréhension et à leur traduction, au Moyen Âge comme à notre époque. Plus que tout autre, ce massif textuel nous paraît refléter les principaux problèmes que rencontre toute entreprise de translation, à savoir la difficulté de rendre le particulier par de l'universel, de transformer du subjectif en objectif, ou encore d'intégrer une nouveauté exogène dans une tradition linguistique, malgré la créativité du latin médiéval désormais largement reconnue ${ }^{1}$.

Pour tenter de donner une idée des écueils spécifiques rencontrés par les traducteurs de ces textes spécialisés au Moyen Âge comme par la suite, j'élirai trois observatoires. La littérature décrivant le monde végétal et animal, que l'on peut qualifier par commodité de naturaliste,

1. Sur ces thèmes, voir notamment M. Parisse, M. Goullet éd., Les Historiens et le latin médiéval. Colloque tenu à la Sorbonne, septembre 1999, Paris, 2001 ; P. Bourgain, Le Latin médiéval, Turnhout, 2005 (L'atelier du médiéviste, 10); et entre autres titres récents R. Librandi, R. PIro éd., Lo scaffale della biblioteca scientifica in volgare (secoli XII-XVI), Atti del Convegno (Matera, 14-15 ottobre 2004), Florence, 2006 ; M. Goyens, P. De LeEmans, A. SMEt éd., Science translated. Latin and Vernacular Translations of Scientific Treatises in Medieval Europe, Louvain, 2008. 
soulève d'emblée des questions d'identification, donc de compréhension en synchronie : une humble plante locale, observable en un milieu bien particulier, a-t-elle toujours un nom latin ? Le deuxième terrain d'observation sera fourni par les écrits médicaux tentant de rendre compte des signes du corps, notamment par le prisme des couleurs : des dénominations venues d'ailleurs trouvent-elles forcément un équivalent dans la langue cible ? Les nuances colorées sont-elles les mêmes pour tous les yeux de l'époque et les nôtres ? Cette incursion dans le domaine de la médecine, notamment de la science des urines qui soulève la question de la dénomination des couleurs et de leur traduction, nous mettra en présence de phénomènes de résistance, d'adaptation ou de création. Avec les traités relatifs à la science des astres, enfin, largement nourrie à partir du $\mathrm{XII}^{\mathrm{e}}$ siècle de textes d'origine arabe ou grecque, on retrouvera certains de ces aspects, encore accrus par le fait que certains termes ont pu se révéler intraduisibles, rebelles à toute tentative d'intégration, sauf au prix de néologismes.

C'est donc à nouveaux frais que je souhaite revenir ici sur une gamme de textes qui m' ont beaucoup occupée, à savoir le Liber subtilitatum de Hildegarde de Bingen (1098-1179), enfin disponible dans des éditions critiques $^{2}$ venues supplanter celle de Migne sur laquelle était fondée l'unique traduction française de cette œuvre ${ }^{3}$, les traités sur les urines, et les traités d'astrologie. Au gré de ces sondages visant à montrer quelques aspects de la difficulté de "dire presque la même chose », selon la formule d'Umberto $\mathrm{Eco}^{4}$, et les choix ou les renoncements des traducteurs, on se posera aussi la question de la possibilité de traduire ces textes de nos jours.

\section{Faire passer le monde végétal d'une aire linguistique à une autre}

Le Liber subtilitatum diversarum naturarum creaturarum composé entre 1150 et 1158 par Hildegarde de Bingen selon ses propres dires, et rebaptisé Physica par son premier éditeur, Jean Schott ${ }^{5}$, est une encyclopédie

2. Hildegard von Bingen, Physica. Edition der Florentiner Handschrift (Cod. Laur. Ashb. 1323, ca. 1300) im Vergleich mit der Textkonstitution der Patrologia Latina (Migne), éd. I. Müller, C. Schulze, Hildesheim, 2008 ; EAD., Physica. Liber subtilitatum diversarum naturarum creaturarum, Textkritische Ausgabe, éd. R. Hildebrandt, T. Gloning, Berlin/ New York, 2010, 3 vol. On privilégiera ici cette dernière édition.

3. Hildegarde de Bingen, Le Livre des subtilités des créatures divines (Physique), trad. P. Monat, Grenoble, 1988-89, 2 vol.

4. U. Eco, Dire presque la même chose. Expériences de traduction, trad. fr. Paris, 2007.

5. Physica s. Hildegardis. Elementorum, Fluminum aliquot Germaniae, Metallorum, Leguminum, Fructuum et Herbarum : Arborum et Arbustorum : Piscium denique, Volatilium et Animantium terrae naturas et operationes IV libris mirabili experientia posteritati tradens, Strasbourg, 1533. Ce titre et cette organisation de l'œuvre ne se trouvent dans aucun manuscrit et il est probable que le Liber subtilitatum originel ait subi après la mort de son auteur un double destin, une scission en deux écrits distincts et des ajouts ; j'ai tenté de raconter cette 
naturelle fondée sur l'œuvre des Six jours mais fortement ancrée dans une réalité locale. Non seulement la représentation du monde naturel, mais aussi celle d'un environnement particulier, en l'occurrence les bords du Rhin dans le Palatinat, font qu'on a pu saluer Hildegarde comme une naturaliste pionnière dans le monde latin ${ }^{6}$, évoquant pour la première fois telle ou telle plante ou espèce ${ }^{7}$, et donc un témoin de premier ordre pour qui tente de reconstituer l'histoire de l'environnement dans le monde germanique au $\mathrm{XII}^{\mathrm{e}}$ siècle. Cette œuvre est en outre émaillée de termes vernaculaires, qui sont parfois soit des hapax soit des primeurs. Elle a connu enfin une diffusion limitée mais intéressante : intégrale ou sous forme de fragments, en latin ou en allemand, ces excerpta étant parfois intégrés à leur tour à des œuvres diverses, notamment à un livre de cuisine ${ }^{8}$ et à un herbier au $X^{\mathrm{e}}$ siècle $^{9}$. Cette œuvre nous servira de point de départ pour appréhender les rapports entre l'universel, théoriquement incarné par le latin, et le particulier, prenant la forme de la langue vulgaire, par nécessité ou par choix. On se penchera sur le Liber subtilitatum dans ses deux états, complet et partiel, pour tenter de voir si tout est traduisible dans ce que Hildegarde mentionne, comment les auteurs qui lui ont succédé ont pu lever les obstacles liés à l'irréductibilité des objets locaux qu'elle décrivait, et comment nous, aux $\mathrm{XX}^{\mathrm{e}} \mathrm{Ou} \mathrm{XXI}^{\mathrm{e}}$ siècles, pouvons le cas échéant lever les obstacles à la traduction fidèle du savoir qu'elle voulait transmettre ${ }^{10}$.

Commençons par les leçons qu'on peut tirer, pour le problème plus large de la traduction d'un vocabulaire spécialisé et local, de la tradition fragmentaire du Liber subtilitatum : on en connaît à ce jour quatre témoins

histoire dans l'ouvrage tiré de ma thèse (Le manuscrit perdu à Strasbourg. Enquête sur l'œuvre scientifique de Hildegarde, Paris/Saint-Denis, 1995), puis dans mon introduction à l'édition critique du Cause et cure (Beate Hildegardis Cause et cure, edidit L. Moulinier, recognovit R. BERNDT, Berlin, 2003).

6. Voir H.FISCHER, «Die heilige Hildegard von Bingen, die erste deutsche Naturforscherin und Ärztin », Münchner Beiträge zur Geschichte und Literatur der Naturwissenschaften und Medizin, 7/8 (1929), p. 381-538.

7. Ainsi d'un poisson qui est sans doute la bouvière : voir L. GeISENHEYNER, « Die älteste Nachricht über den Bitterling », Zoologischer Beobachter, 52 (1911), p. 10-12.

8. Voir M. Weiss-Amer, "Die "Physica" Hidegards von Bingen als Quelle für das "Kochbuch Meister Eberhards" », Sudhoffs Archiv, 76/1 (1992), p. 87-96 ; et avant elle, A. FeYL, Das Kochbuch Meister Eberhards. Ein Beitrag zur altdeutschen Fachliteratur, Diss. Freiburg i.Br., 1963.

9. Voir ainsi B. Fehringer, Das "Speyerer Kräuterbuch" mit den Heilpflanzen Hildegards von Bingen, Eine Studie zur mittelhochdeutschen "Physica". Rezeption mit kritischer Ausgabe des Textes, Würzbourg, 1994 (Würzburger medizinhistorische Forschungen, Beiheft, 2). Dans cet « herbier de Spire», des passages entiers de la Physica traduits en allemand côtoient d'autres textes, comme le Deutscher Macer.

10. Entre autres publications collectives sur cette question qu'il n'est pas possible de toutes citer, on verra avec profit O. BERTRAND, H. GeRnER, B. STRUMPF, Lexiques scientifiques et techniques. Constitution et approche historique, Palaiseau, 2007 ; P. DurIs éd., Traduire la science hier et aujourd'hui, Pessac, 2008. 
allemands, du $\mathrm{Xv}^{\mathrm{e}}$ siècle pour la plupart, et cinq témoins latins, dont trois découverts par mes soins à la Bibliothèque vaticane ${ }^{11}$, l'existence de ces fragments impliquant bien évidemment que l'œuvre originelle a été comprise. Encore faut-il préciser que, comme l'œuvre qu'ils démarquent, ces témoins ne sont pas monolingues. C'est ce qu'on appréhendera en se fondant sur le ms. Città del Vaticano, BAV, Pal. lat. 1216, un recueil médical latin-allemand ayant appartenu au comte palatin Ottheinrich (1502-1559) ${ }^{12}$.

La première partie de ce recueil (f. 1-107) date de la fin du XIV ${ }^{\mathrm{e}}$ siècle, avec des ajouts du $\mathrm{Xv}^{\mathrm{e}}$; la seconde (f. 108-260) daterait quant à elle des années 1445-1480 et proviendrait de la région de Worms. C'est toutefois une main inconnue dans le reste du recueil qui a copié les folios $91 \mathrm{v}$ à $95 \mathrm{r}$, qui se présentent comme un ensemble à la fois cohérent et isolé. Couverts d'une trentaine de lignes en moyenne, ils reproduisent quarante-deux recettes médicales, qui ne sont autres que des extraits du Liber subtilitatum. Un même chapitre a pu fournir plus d'une recette, et l'on reconnaît ici en tout trente-sept chapitres répartis entre six des neuf livres qui constituent l'œuvre originelle : vingt-trois chapitres du livre premier, De plantis, quatre du livre III, De arboribus, cinq du livre IV, De lapidibus, un du livre V, De piscibus, trois du livre VI, De avibus, et un du livre IX, De metallis. Mais l'ordre de succession des recettes nous montre qu'on a affaire à des extraits doublés d'un réagencement : l'ordre suivi ici ne reflète pas celui des différents livres, ni même, à l'intérieur de chaque livre, l'ordre originel des chapitres.

Ce fragment est parsemé de termes vernaculaires, et la lecture du texte permet de se rendre compte que le copiste connaissait le latin sans en maitriser parfaitement la grammaire ; mais on ne saurait pour autant lui imputer de manière certaine le recours à un vocabulaire germanique qui figurait déjà peut-être dans le manuscrit dont il s'inspirait : le latin n'était pas non plus la langue maternelle de Hildegarde et tous les manuscrits complets du Liber subtilitatum connus à ce jour contiennent des termes en langue vulgaire. Ces différentes versions étant nettement postérieures à la date de composition de l'œuvre originale, on peut certes y voir l'intervention de copistes ; toutefois il est très probable que l'abbesse elle-même ait employé ces termes, faute de connaître le nom latin de tous les éléments indigènes qu'elle avait entrepris de décrire, en particulier les plantes.

11. Bern, Burger Bibliothek, cod. 525, $\mathrm{xv}^{\mathrm{e}}$ s., f. 18r-23r ; Freiburg in Brisgau, Universitätsbibliothek, cod. 178a, fin XIV -début XV s. (v. 1390-1400), f. 1-15; Città del Vaticano, BAV, Pal. lat. 1144, $2^{\mathrm{e}} 1 / 2 \mathrm{Xv}^{\mathrm{e}}$ s. (Heidelberg), f. 128v-129r ; Città del Vaticano, BAV, Pal. lat. 1207, xve s. (1425/1427, Heidelberg), f. 64r-65v ; Città del Vaticano, BAV, Pal. lat. 1216, fin XIV s., f. 91v-95.

12. On me permettra de renvoyer à L. Moulinier, «Fragments inédits de la Physica : contribution à l'étude de la transmission des manuscrits scientifiques de Hildegarde de Bingen », Mélanges de l'École française de Rome, 105.2 (1993), p. 629-650. 
De fait, c'est dans le domaine botanique que les emprunts au vulgaire sont ici les plus nombreux : treize noms de végétaux sont traduits, alors que l'équivalent vernaculaire n'est donné que pour trois noms de maladies ( « constrictio guthuris id est strengelen », « dolor capitis id est heubtsucht», et « bubo id est sler»), un verbe (« si pabulum nimis avide sumpsit, quod volgariter dicitur virfangen vel zerese »), et deux noms d'insectes (« eruce id est rupen et papiliones id est cyfelder $\left.{ }^{13} \gg\right)$.

En revanche, dès qu'il s'agit de désigner arbres ou plantes, donc de permettre leur identification, plusieurs cas de figure se présentent :

- le nom latin d'une espèce peut être accompagné de synonymes euxmêmes latins comme pour l'arum (« herba que dicitur aaron id est jarus vel serpentina ») ${ }^{14}$ ou le calendula (« ryngele sive flammula ») : le Liber subtilitatum tel qu'on le connaît dit précisément ringula, et son identification avec la «flamula » est confirmée au Xv ${ }^{\mathrm{e}}$ siècle par le Speyrer Kraüterbuch, qui donne d'autres équivalents possibles en allemand ${ }^{15}$;

- le nom peut aussi être un simple calque sémantique de son nom vernaculaire $^{16}$, comme avec « pentafilon funfblat » (potentille), où la formation $\mathrm{du}$ nom germanique reproduit exactement celle du nom grec signifiant « cinq feuilles », ou « lingua cervina » qui est la traduction littérale de Hirtzunge, « Hirschzunge », scolopendre ${ }^{17}$;

- un nom latin peut également être suivi, après la formule « id est », et c'est le cas le plus fréquent, du nom vulgaire lui correspondant exactement, comme dans les glossaires latins-allemands que les recueils médicaux d'origine germanique comportent la plupart du temps (et on signalera ici qu'un tel glossaire se trouve dans le Pal. lat. 1216) : «esculus id est spirbaum » (sorbier) ; « zizaniam id est rade » (cumin noir ou nigelle cultivée) ; « semperviva id est huswurtz » (joubarbe ${ }^{18}$ ) ; « solatrum id est

13. Rupe a donné l'allemand actuel Raupe, «chenille », et Falter, «papillon », est le descendant du vieil haut-allemand vivaltra, du germanique fifaldron.

14. Le Speyerer Kraüterbuch donne : "Aarona heißet aron[a] oder roder myntze » (p. 69). D'après Hermann Fischer, cette plante était également appelée jarus et serpentaria minor dans le Gart der Gesundheit, version allemande du Hortus sanitatis ( $c f$. H. FISCHER, Mittelalterliche Pflanzenkunde, Munich, 1929, p. 260) ; Jerry Stannard relève pour sa part serpentaria dans une version du XIII ${ }^{\mathrm{e}}$ siècle du Circa instans de Matthaeus Platearius (J. STANNARD, «Identification of the plants described by Albertus Magnus, De vegetabilibus lib. VI », Respublica Litterarum, II [1979], p. 231-318, p. 288, s. v. basilicus). Le nom "Aaron » n'est là que par déformation du latin Arum (cf. G. HeGI, Illustrierte Flora von Mitteleuropa, Munich, 1906, vol. II, p. 132).

15. Speyerer Kraüterbuch, p. 118 : « flamula heisset brynnende krut oder wasser seim ».

16. Sur cette notion, on verra notamment C. Nicolas, Vtraque lingua. Le Calque sémantique : domaine gréco-latin, Paris/Louvain, 1996.

17. Cf. G. Hegr, Illustrierte Flora von Mitteleuropa..., vol. I, p. 34.

18. Le nom linnéen de cette plante est « sempervivum tectorum », et son nom germanique rappelle sinon la toiture, du moins la maison. 
nachtschade » (morelle noire) ; « tanacetum id est reynevane » (tanaisie) ; « rustica id est byverwurz » (aristoloche) ;

- enfin, le nom latin peut manquer purement et simplement, comme dans le cas du lierre (« herba que dicitur gunderebe »); de l'épurge (« herba que brachwurtz dicitur »); de l'athamante (« herba que dicitur berwurz »); ou encore de la sariette (« herba que dicitur peffircrut »), si c'est bien d'elle qu'il s'agit ${ }^{19}$.

Brisons là avec ce fragment que l'on a cité au-delà de son intérêt pour la recherche hildegardienne, afin de donner un aperçu des impasses ou des choix liés à la traduction d'un vocabulaire spécialisé et géographiquement ancré, en ouvrant notre réflexion au monde animal et à ses mots.

\section{Le secours des lexiques}

Élargissons l'enquête au Liber subtilitatum intégral et non plus à ses excerpta, afin d'apprécier, en synchronie comme en diachronie, les problèmes posés par l'interprétation et la traduction du savoir naturaliste qu'il véhicule. On l'a dit, c'est souvent sous un nom local que sont présentées des espèces et on doit ainsi à Hildegarde un témoignage de premier plan sur le monde végétal et animal connu en Germanie au XII ${ }^{\mathrm{e}}$ siècle. Mais certaines appellations ne se laissent pas aisément résoudre, et souvent plusieurs identifications sont possibles car l'abbesse, préférant manifestement les subtilitates des choses à leur apparence physique, ne livre aucune description, ni des plantes ni des animaux, dont elle est pourtant capable de rapporter certains traits ou comportements, comme le frai dans le cas des poissons.

Une première difficulté de taille a pu être constituée par les erreurs des copistes, estropiant les noms et rendant inidentifiables les créatures désignées. Un poisson nommé welca apparaît ainsi dans le manuscrit Paris, BnF, lat. 6952 qui a servi à l'édition de Migne, mais il s'agit d'une faute : le manuscrit Firenze, Biblioteca Medicea Laurenziana, Ashburnham 1323 dit welra, et il faut y voir le silure glâne, « poisson-chat géant » pouvant atteindre $300 \mathrm{~kg}$ dans le Danube. Au XIII ${ }^{\mathrm{e}}$ siècle, Albert le Grand témoigne de la survie de l'appellation locale : « et aput nos talis piscis est cuius venter est magnus et stomachus villosus omni genere villorum, qui aput nos welre vocatur $^{20} \gg$.

Le Ruhlheubt du manuscrit de Paris est une autre faute de copiste, comme on s'en rend compte en comparant avec le culhoubit du Summarium

19. Pierre Monat rend peffercrut par «cresson » (Hildegarde De Bingen, Le Livre des subtilités des créatures divines, vol. I, p. 64). Danielle Delley lui préfère «sariette », Satureia montana (D. Delley, Hildegarde de Bingen et les plantes médicinales, Bâle, 1988, p. 58).

20. «Et chez nous il y a un poisson dont le ventre est grand et l'estomac tapissé de toutes sortes de poils, que l'on appelle welre » : voir Albertus Magnus de animalibus libri XXVI, nach der Cölner Urschrift, éd. H. STADLER, Münster, 1916, p. 263. 
Heinrici; au XVI ${ }^{\mathrm{e}}$ siècle, Conrad Gesner explique les différentes appellations de cet animal à grosse tête par le radical caput dans les langues romanes et haupt en allemand : "gallice Chabot [...] Germanice Kulheit/Kaulheupt, etc. Ex his nominibus pleraque a capitis magnitudine facta sunt, alia ab ejusdem rotunditate $^{21} »$.

Meysisch est aussi une erreur du scribe : il faut lire meyfisch, grande alose, poisson marin qui vient déposer ses œufs en rivière, et remontait autrefois le Rhin jusqu'en amont de Bâle.

Enfin, si nous quittons le monde des poissons pour d'autres espèces, on se rend compte qu'oiseaux et amphibiens n'ont pas été épargnés par des erreurs vouant $a$ priori à l'échec toute tentative de traduction fidèle. Le témoignage postérieur de Conrad Gesner nous permet ainsi de reconnaître en l'animal appelé à tort Scherzbedra la tortue bourbeuse, aujourd'hui disparue de la plaine rhénane ; ces lignes du grand naturaliste suisse évoquant le scherzenfider ne laissent en effet pas place au doute quant à une cacographie du copiste : «Germani Schiltkrot vel Tallerkrot id est rubetam testa scuti aut orbis effigie intectam [...]. Aliqui Germanice scherzenfider nimirum quod pedes earum quodammodo alas referant, praecipue marinarum ${ }^{22}$. »

Quant à l'oiseau appelé ici beynstercza, il apparaît, à nouveau grâce au témoignage de Gesner, que c'est celui que Hildegarde avait dû nommer Begesterz ou Wegesterz avant les déformations dues aux scribes : « De Motacilla quam nostri albam cognominant germanice appellatur ein wysse oder grawe wasserstelz oder bachstelz : quiksterz id est alba aut fusca motacilla, a s. Hildegardi Begesterz vel Wegesterz, avis temperamentum illa calidum et humidum esse scribit ${ }^{23}$.

Au livre des reptiles, le chapitre Harumna est lui aussi problématique car les différentes sources nous renseignent sur des formes voisines mais pas exactement sur le terme employé par Hildegarde. Le Summarium Heinrici pose : «migale quasi gulosus harmo », et dans le glossaire latinallemand qui se trouve à la fin du manuscrit Ashburnham 1323, on lit : « megalus harmel/ranunculus herume ». Le manuscrit Città del Vaticano,

21. «En français Chabot, en allemand Kulheit/Kaulheupt, etc. Parmi ces noms, la plupart ont été forgés d'après la taille de la tête [du poisson], d'autres d'après sa forme ronde » (Icones animalium quadrupedum viviparum et oviparum quae in Historia animalium Gesneri libro I et II describuntur, editio secunda, Zurich, 1560, p. 291).

22. «Les Allemands disent Schilkrot ou Tallerkrot, c'est-à-dire "crapaud recouvert de la carapace d'un bouclier ou de l'image d'un cercle" [...]. D'autres disent Scherzenfider en allemand, sans doute parce qu'ils rapportent leurs pattes à des ailes, surtout celles des [tortues] marines » (NdT : fider signifie plume) ; voir Gesneri medici Tigurini Historiae animalium libri IV, Zurich, 1551-1558, Historiae animalium lib. IIII de quadrupedibus oviparis, p. 91.

23. "Le hoche-queue que chez nous on appelle blanc, les Allemands le nomment ein wysse oder grawe wasserstelz, ou bachstelz: le quiksterz, c'est-à-dire le hoche-queue blanc ou brun, a un tempérament chaud et humide selon ce qu'écrit sainte Hildegarde » (C. Gesneri medici Tigurini Historiae animalium libri IV, Historiae animalium lib. III qui est de avium natura, p. 593). 
BAV, Reg. lat. 1701, étudié par E. Steinmeyer et E. Sievers, comporte pour sa part migalus haremo ${ }^{24}$, et enfin Albert évoque un animal nommé haren ${ }^{25}$; de ces différents indices, il ressort que Harumna pourrait être le crapaud sonneur, rendu méconnaissable par une faute de copie ${ }^{26}$.

À la recherche de l'identité de plantes ou d'animaux, préalable indispensable à toute entreprise de traduction, on peut donc tirer parti d'éclairages rétrospectifs : outre la colossale somme de connaissances rassemblée par Conrad Gesner, «prince des zoologues » ayant lu tous ses prédécesseurs selon Robert Delort ${ }^{27}$, on ne saurait évidemment négliger l'apport du De animalibus d'Albert le Grand qui cite à son tour nombre d'animaux sous leur nom allemand ${ }^{28}$. Un exemple éclairant du fruit qu'il y a à tirer de rapprochements rétrospectifs peut être fourni par la mention de l'animal appelé Merkacza traduit à tort par «phoque » dans le dictionnaire de Franz Blatt, qui comprenait ce terme comme " chat de mer » et non pas comme «chat d'outre-mer $»^{29}$. Il s'agit en fait du macaque ou magot de Gibraltar, Macaca sylvanus L., dont la ressemblance avec le chat est évoquée entre autres par le Ruodlieb et par Albert le Grand ${ }^{30}$, et dont la présence effective en Allemagne au temps de Hildegarde peut être inférée du constat que fait Gesner au XvI e siècle : «Advehuntur aliquando in Germania cercopitheci parvi (kleine Meerkatzen) barba caput totum ambiente ${ }^{31}$.»

Il est bien sûr encore plus fructueux de mener une enquête lexicographique dans des sources proches dans le temps comme dans l'espace du Liber subtilitatum de Hildegarde. La comparaison avec le Ruodlieb, qui énumère dix-huit espèces différentes présentées comme des poissons

24. Voir E. Steinmeyer, E. Sievers, Die althochdeutschen Glossen, Berlin, 1879-1895, 3 vol., vol. III, p. 453.

25. Alberti Magni Opera omnia, éd. A. Borgnet, Paris, 1890-1899, 38 vol., vol. XII, p. 555 : «Semerion philosophus dicit esse serpentem de genere draconum marinorum : et concordat cum eo Avicenna. [...] et est niger aliquantulum pilosus juxta caput et facit vulnus in morsu magnum et malum : et accidit ex morsu suo quod accidit ex morsu viperarum. »

26. Pour d'autres exemples, voir L. Moulinier, « La faune germanique médiévale aux $\mathrm{XI}^{\mathrm{e}}$-XII ${ }^{\mathrm{e}}$ siècles : une brève histoire de noms », dans F. MorenzonI et É. Mornet éd., Milieux naturels, espaces sociaux. Études offertes à Robert Delort, Paris, 1997, p. 193-208.

27. R. Delort, Les Animaux ont une histoire, Paris, 1984, p. 47.

28. Entre de nombreux exemples, voir par exemple ce qu'Albert dit du nom de la lotte, alroppa selon Hildegarde, ruppa dans les Constitutiones Hirsaugienses, rupba dans le Summarium Heinrici : « qui aput nos vocatur alrute et quidam vocant ipsam alrupe ; quidam autem simpliciter ruptam vocant » (Albertus Magnus de animalibus libri XXVI, p. 268).

29. F. BLatt, Novum Glossarium, Copenhague, 1959, s.v.

30. Albertus Magnus de animalibus libri XXVI, p. 1422-1423 : « corpori videtur ex symia et catto agresti, cum vultum simiae habet » («Quant à son corps, on dirait un chat sauvage et un singe, et il a un visage de singe »).

31. "On amène parfois en Allemagne de petits cercopithèques dont la tête est entourée d'une barbe (de petits macaques) » : cf. C. Gesneri medici Tigurini Historiae animalium libri IV, Historiae animalium lib. I de quadrupedibus viviparis, p. 970. 
pêchés localement ${ }^{32}$, ou les Constitutiones Hirsaugienses attribuées à l'abbé Guillaume de Hirsau ${ }^{33}$, dont une large partie est consacrée au langage des signes à utiliser dans la vie quotidienne pour respecter la règle du silence, a confirmé mainte identification, sans oublier le Summarium Heinrici, sorte de compendium du savoir largement fondé sur les Étymologies d'Isidore de Séville qui vit le jour vers 1100 dans la région de Worms ${ }^{34}$. Mais il faut aussi souligner avec force la moisson que l'on peut tirer de l'étude des glossaires ou synonyma anonymes et souvent inédits, notamment des glossaires latin-allemand contenus dans des codices comme le manuscrit Città del Vaticano, BAV, Pal. lat. 1414 (XIII ${ }^{\mathrm{e}}-\mathrm{XIV}^{\mathrm{e}}$ siècle), qui énumère trente-huit noms de poissons présents dans la Moselle au Moyen Âge, ou le manuscrit BAV, Pal. lat. 1382 (copié dans le Sud-Ouest de l'Allemagne dans la seconde moitié du XIV ${ }^{\mathrm{e}}$ siècle) ${ }^{35}$.

De tels glossaires offrent au moins autant de ressources pour qui cherche à cerner l'identité de végétaux : le chapitre 37 du liber de plantis, par exemple, est consacré à une plante appelée " sisimera $^{36}$ », terme que le traducteur moderne a rendu en le francisant en "sysémère ${ }^{37}$ »; or différents éclairages permettent d'approcher d'une identification précise et de comprendre qu'il s'agit d'un type de menthe, la balsamite, à commencer par la leçon du Summarium Heinrici : «sisimbrium sisimra vel tenalis vel

32. Ruodlieb, der älteste Roman des Mittelalters, éd. F. SEILER, Halle, 1882.

33. Sancti Willhelmi constitutiones Hirsaugienses seu Gengenbacenses, éd. J.-P. Migne, PL 150, col. 923-1146.

34. Sur les rapports possibles entre cette œuvre et les connaissances de Hildegarde, voir R. HiLdebrandT, « Summarium Heinrici : Das Lehrbuch der Hildegard von Bingen », dans E. Bremer, R. Hildebrandt éd., Stand und Aufgaben der deutschen Dialektlexikographie, Berlin/New York, 1996, p. 89-110.

35. À la toute fin $\mathrm{du} \mathrm{xx}^{\mathrm{e}}$ siècle, un important colloque avait attiré l'attention sur la richesse de ce type de sources : J. HAMESSE, D. JACQUART éd., Lexiques bilingues dans les domaines philosophique et scientifique (Moyen Âge-Renaissance). Actes du colloque international organisé par l'École pratique des hautes études-IVe Section et l'Institut supérieur de philosophie de l'Université catholique de Louvain, Paris, 12-14 juin 1997, Turnhout, 2001. Plus récemment, voir J. Hamesse et J. Meirinhos éd., Glossaires et lexiques médiévaux inédits. Bilan et perspectives, Porto, 2011.

36. Hildegard von Bingen, Physica. Liber subtilitatum diversarum naturarum creaturarum, vol. I, p. 79 : «sisimera calida existit. Et qui venenum comedit aut bibit, accipiat sisimeram et rutham atque batheniam equali pondere, et in mortario tunsis succum eorum exprimat, et tunc etiam de succo sprincwrtz bis tantum sumat ut unius istorum est, et predicto succo addat, et simul conmisceat, ac ita per pannum colet, et ieiunus bibat » [les caractères en gras sont des éditeurs].

37. Hildegarde De Bingen, Le Livre des subtilités des créatures divines, trad. P. Monat, Grenoble, 2 vol., 1989, vol. I, p. 63 : « La sysémère est chaude. Si on a mangé ou bu du poison, prendre de la sysémère, de la rue et de la bétoine en poids égaux, piler dans un mortier pour exprimer le suc; ajouter deux fois autant de suc de saponaire, mélanger, filtrer avec un linge et boire à jeun. » NdT : «J'ai francisé le mot latin utilisé par Hildegarde. Il semble désigner ce qu'on appelle en France les Fils de la Vierge. » 
menta veneria vel balsamita ${ }^{38} »$. Et cette identification est confirmée par un manuscrit comme le manuscrit Città del Vaticano, BAV, Vat. lat. 4482, f. 68v (« sinsibrium vel sistinbruom id est balsamita »), ou encore par une glose d'un des manuscrits du Liber subtilitatum (BML, Ashb. 1323, cap. 178).

Un autre problème d'identification est posé par le terme de « Wizsgras ${ }^{39}$ », que la traduction française rend par le générique « herbes des prés ${ }^{40} »$; là encore, la consultation de manuscrits, en l'occurrence plus tardifs, peut aider à affiner l'identification : un herbier comme celui de Hieronymus Harder (1523-1607), conservé dans le ms. Città del Vaticano, BAV, Pal. lat. 1276, donne au f. 125r « Poligonium Weggraß ${ }^{41}$ », de même que le ms. BAV, Pal. lat. 1256 ( $\mathrm{Xv}^{\mathrm{e}}$ siècle), avec ses Synonyma apothecariorum et ses Synonyma simpliciumen en latin et en allemand, m'a permis notamment d'identifier une autre plante mystérieuse nommée par l'abbesse, le pfriselouch, glosé en porrula au f. 208va.

Concluons cet échantillonnage du Liber subtilitatum et ce passage en revue des problèmes posés par la traduction de noms d'espèces et des ressources qui s'offrent au traducteur en évoquant l'infiniment petit, le monde des insectes, qui ne portent pas encore ce nom là au XII ${ }^{\mathrm{e}}$ siècle. Dans un chapitre du Liber de plantis consacré à la grande menthe, il est question de suren ou de sneuelzen qui blessent l'homme en le mangeant ${ }^{42}$, et le traducteur a choisi de laisser le second terme intact dans la version française de ce passage, le glosant dans une note en « parasite non identifié » : «Il y a une autre menthe, qui est grande et plus chaude que froide. La piler. Lorsque des vermines, ou des snebelcz blessent l'homme en le dévorant, en attacher par-dessus avec un linge, et ils mourront ${ }^{43}$. » Or ce terme n'est pas intraduisible, et il figure dans un des manuscrits dépouillés par

38. Summarium Heinrici, éd. R. Hildebrandt, 2 vol., Bd. 2, Textkritische Ausgabe der 2. Fassung Buch I-VI sowie des Buches XI in Kurz und Langfassung, Berlin/New York, 1982, p. 56.

39. Hildegard von Bingen, Physica. Liber subtilitatum diversarum naturarum creaturarum, I, 85, vol. I, p. 103 : «Wizgras (Weggraß) et slizgras (suregraß) et romesse gras temperate nature et temperate succi sunt...»

40. Hildegarde de Bingen, Le Livre des subtilités des créatures divines, vol. I, p. 106 : «Ces herbes des prés sont de nature tempérée, et modérément sèches. »

41. Voir J. MonTEBAur, «Ein süddeutsches Herbarium in der Vatikanischen Bibliothek », Archiv für Geschichte der Mathematik, der Naturwissenschaften und der Technik, 11 (1929), p. 271-276.

42. Hildegard vON BINGen, Physica. Liber subtilitatum diversarum naturarum creaturarum, I, 76, « De Myntza maiori », vol. I, p. 100 : « Alia minza que magna est, calida magis quam frigida. Ista contundatur, et ubi suren aut sneuelzen hominem comedendo ledunt, illuc circa desuper ponatur et panno ligetur et morientur. »

43. Hildegarde de Bingen, Le Livre des subtilités des créatures divines, vol. I, p. 110. 
E. Steinmeyer et E. Sievers assorti d'un équivalent latin, scinifes, qui invite à y voir une sorte de cousin ${ }^{44}$.

Le recours aux glossaires que renferment de nombreux manuscrits a donc pu être une aide précieuse pour les lecteurs médiévaux confrontés à un vocabulaire non pas tant spécialisé que localisé, et on peut s'appuyer encore sur eux de nos jours pour comprendre et traduire correctement certaines réalités du Moyen Âge. Certains ont fait l'objet d'éditions, tels les Sinonima Bartholomaei composés en Angleterre ${ }^{45}$, ou l'Alphita, un lexique botanicomédical originaire d'Italie daté des dernières années du XII siècle ou des premières du XIII , et on ne peut que souhaiter la poursuite du mouvement malgré les difficultés de ce genre d'entreprise ${ }^{46}$. Demandons-nous pour finir si tout était susceptible d'explication par la périphrase ou le synonyme en langues vernaculaires, ou si au contraire il y a de l'intraduisible dans la Fachliteratur médiévale. C'est ce dont j'essaierai de donner un aperçu par une incursion dans deux autres domaines de la science d'alors, la médecine et la science des étoiles.

\section{Les problèmes liés à la traduction des couleurs}

Rappelons très vite le rôle central de l'examen des urines dans la consultation médicale au Moyen Âge, phénomène sur lequel je me suis abondamment exprimée ailleurs ${ }^{47}$. On imaginait à l'intérieur du corps un processus de coction en plusieurs étapes, et le degré de cuisson était rendu manifeste par la couleur de la substance ainsi « cuite ${ }^{48}$. Aux différentes teintes de l'urine était attribuée une signification relative aux qualités élémentaires et aux humeurs, et ce mode de lecture du corps prit un nouvel essor en Occident après la traduction en latin, à la fin du $\mathrm{XI}^{\mathrm{e}}$ siècle, du Peri ouron du Byzantin Théophile. Cet ouvrage introduisait un nuancier des couleurs de l'urine que ses successeurs latins reprirent et enrichirent pour fixer la gamme de ces teintes en un nombre oscillant entre dix-neuf et vingt, la couleur noire

44. E. Steinmeyer, E. Sievers, Die althochdeutschen Glossen, vol. 3, p. 452 : « Codex selestadiensis f. 110 a : scinifes snebilazir. »

45. Sinonima Bartholomei, A glossary from a fourteenth-century manuscript in the Library of Pembroke College, Oxford, éd. J. L. G. Mowat, Oxford, 1882.

46. Voir Alphita, éd. S. De Renzi, dans Collectio Salernitana, 5 vol., Naples, 18521859, vol. III, p. 271-322, édition fondée sur un seul manuscrit ; Alphita. A medico-botanical glossary, from the Bodleian manuscript, Selden B. 35, éd. J. L. G. MowAT, Oxford, 1887, qui édite un texte incomplet. Il faut désormais leur préférer A. GARCía GonZaLEZ éd. et comm., Alphita, Florence, 2007.

47. On me pardonnera de renvoyer à L. Moulinier-Brogi, L'Uroscopie au Moyen Âge. «Lire dans un verre la nature de l'homme », Paris, 2012.

48. Pour un résumé de ces théories, voir F. WALLis, « Signs and Senses : Diagnosis and Prognosis in Early Medieval Pulse and Urine Texts », dans P. Horden, E. Savage-Smith éd., The Year 1000 : Medical Practice at the End of the First Millennium, Social History of Medicine, 13 (2000), p. 265-278 (p. 268). 
pouvant être comptée deux fois, selon qu'elle était précédée de la couleur verte, ou de la nuance livide. Mais le transfert ne se fit pas sans difficultés pour toutes les désignations chromatiques.

En matière de noms de couleurs, l'uroscopie a enrichi la langue latine dans deux directions : en forgeant des néologismes, tel subcitrinus, " citrine pâle ${ }^{49}$ », et en y introduisant tels quels des mots grecs empruntés à Théophile pour lesquels le latin manquait d'un équivalent exact. Commençons par la créativité du latin médiéval : dans la gamme canonique des couleurs de l'urine qui se met en place à la fin du XII siècle, certaines teintes sont caractérisées par rapprochement avec une autre (ainsi rouge pâle ou rougeâtre, roux pâle ou roussâtre, etc.), en général grâce au préfixe sub-, lui-même héritier du grec hypo-, qui se trouvait déjà chez Homère pour les termes chromatiques. Trois termes de couleur furent en revanche directement pris à Théophile, soit faute d'équivalent, comme pour inopos, « rouge vineux », ou charopos, « gris cendré », soit parce que le mot avait dans la langue source un autre type d'emploi, comme c'est le cas pour kyanos, « rouge tirant sur le bleu, pourpre ${ }^{50}$ ».

Or ces trois termes, qui avaient résisté à toute traduction latine et furent intégrés à la langue cible dans leur extranéité, sous forme invariable, ont souvent à leur tour été laissés tels quels par les passeurs qui, à partir du $\mathrm{XIII}^{\mathrm{e}}$ siècle, tentèrent de transférer le savoir uroscopique dans différentes langues vulgaires.

Dans un manuscrit conservé à Rome à l'Accademia dei Lincei (43 B 28) et contenant une version italienne des Regulae urinarum de Maurus de Salerne ( $\dagger 1214)$, on trouve bien dix-neuf teintes, mais les désignations de couleur héritées du grec ont été reproduites telles quelles (si l'on excepte une modification graphique pour kyanos devenu quianos). Impossibilité de traduire ou désir de garder intactes des notions-clés, avec pour ainsi dire leur marque d'origine ? On pencherait plutôt pour la seconde hypothèse, d'une part parce que la langue italienne dispose du mot « ciano » à partir $\mathrm{du} \mathrm{Xv}^{\mathrm{e}}$ siècle pour désigner un type de bleu, comme l'atteste la Practica de Michel Savonarole ( rubeus pulverulentulus est, qui assimilatur sanguini putrefacto extracto ex vena, quem alii kiano appellaverunt $\left.{ }^{51} \gg\right)$, et d'autre part parce que le traducteur anonyme n'est pas en peine d'italianiser ailleurs

49. Les Regulae urinarum de Maurus sont le seul exemple cité pour le vocable " subcitrinus » par M. RoQues, dans ses Additions et corrections d'Antoine Thomas au Glossaire de Du Cange, Bruxelles, 1952, p. 149. Or on le trouve par exemple dans le De Urinis attribué à Mattheus de Archiepiscopo : voir Collectio Salernitana, éd. S. De RenzI, t. IV, p. 506-512 (p. 507).

50. Le latin classique ne disposait de toute façon pas d'un mot précis pour dire «bleu»: voir notamment M. PAStoureau, Bleu, histoire d'une couleur, Paris, 2000.

51. Michaelis Savonarole de urinis summa, dans Practica canonica de febribus Io. Michaelis Savonarole, eiusdem de pulsibus, urinis, egestionibus, vermibus, balneis, Venise, 1552 , f. 112 v. 
des termes certes latins, mais directement hérités du grec comme les noms des fièvres ${ }^{52}$. On connaît par ailleurs au $\mathrm{XV}^{\mathrm{e}}$ siècle l'existence d'équivalents vernaculaires pour karopos : dès l'année 1429, le terme est attesté dans la Chronique de Morosini, et, au milieu du siècle, Michel Savonarole en donne un équivalent en vulgaire ${ }^{53}$.

Le langage des couleurs de l'urine a donc pu soulever des difficultés lors du passage du latin au vulgaire, mais parfois aussi, hors de toute optique de traduction, offrir des obstacles à la compréhension, comme le suggère une « roue des urines » conservée dans le manuscrit København, Det Kongelige Bibliotek, NKS Fol. 84b ; elle y figure seule, indépendamment de tout texte, au folio 4, et présente une absence significative : le terme d'inopos ne figure pas à sa place dans la légende, ce qui reflète peut-être un doute quant à sa signification - un doute dû à l'ignorance de l'origine grecque du mot, oinopos, « vineux », « couleur de vin ».

Ces limites au transfert de savoir en matière uroscopique s'expliquent sans doute par la grande difficulté qu'il y a à éliminer la part de subjectivité dans ce domaine de la dénomination linguistique. Dans les listes de couleurs d'urines qui se transmettaient en Occident depuis le $\mathrm{XII}^{\mathrm{e}}$ siècle, chaque couleur était désignée par un adjectif que les auteurs explicitaient en recourant à des comparaisons, reflétant elles-mêmes des conditions géographiques et linguistiques précises : si la couleur du chou ou du poireau ne pose pas de difficultés, il entre en revanche une part de subjectivité ou d'imprécision dans des comparaisons avec une corne de telle ou telle teinte, une viande à demi-cuite, des châtaignes bien mûres ou encore un vin trouble et gâté, sans parler du parallèle avec les poils de chameau ou de lion qui ne se trouvent pas sous tous les climats. Et quand Michel Savonarole, dans l'Italie de la seconde moitié du $\mathrm{XV}^{\mathrm{e}}$ siècle, donne à son tour des explications sur les couleurs des urines, il fait appel à des objets qui ne pouvaient être aussi familiers à tous ses prédécesseurs ou contemporains, comme l'orange, la cerise, le safran ou l'écarlate ${ }^{54}$.

52. « Sinochus » devient « sinoco », « epigmasticus » devient « et pimasticus » (mais « epimastico » dans la rubrique), « augmasticus » est rendu par « aumastico » (mais « augmastico » dans la rubrique), et « homotenus » par « homoteno » (« homotano » dans la rubrique). «Causon», en revanche (un mot désignant une fièvre ardente), n'a pas ici d'équivalent italien ; pour plus de détails, voir L. Moulinier, « L'uroscopie en vulgaire dans l'Occident médiéval : un tour d'horizon », dans M. Goyens, P. De Leemans, A. Smet éd. Science translated..., p. 221-241.

53. Michaelis Savonarole de urinis summa..., f. $112 \mathrm{v}:$ : in vulgari nostro dicitur beretin ».

54. De urinis summa, p. 661-662: «Citrinus, intensioris citrinitatis est, qui est intensior citrino, ut est metrum, et assimilatur pomo arancii non valde citrini » [...]. « Rufus, qui est valde citrinus, tendens ad aliquam albedinem, cuiusmodi est color ceresi dulcis et maturi » [...]. « Postea est rubeus clarus, qui assimilatur rosae rubeae clarae valde, ut est scarlata. » 
Les objets pris comme points de comparaison et les adjectifs employés dans les traités d'uroscopie pouvaient donc poser problème, qu'il s'agisse de les interpréter ou, surtout, de les traduire, sans qu'une règle unique d'action se dégage. Dans un Livre des orines en français du $\mathrm{XIV}^{\mathrm{e}}$ siècle conservé à Londres ${ }^{55}$, par exemple, la plupart des comparaisons des traités d'uroscopie latins sont conservées, alors que les adjectifs de couleur sont pour beaucoup laissés tels quels : or la langue vernaculaire disposait d'équivalents, tel « sozpale » pour «subpallidus », attesté dans le même codex, folio 215, au sein d'une traduction française du De adventu medici ad aegrotum salernitain ${ }^{56}$.

Et dans le dernier chapitre de cet opuscule, plusieurs phénomènes intéressant l'histoire des traductions se donnent à voir : le décalque pur et simple de comparaisons invoquant des réalités exogènes, comme le poil de chameau ou le safran d'Orient, le maintien de termes dans leur langue d'origine, en l'occurrence le latin et le grec, l'introduction de comparants indigènes, comme le safran « de cortil » ou la garance, et le traitement inégal réservé aux adjectifs de couleur issus du grec : karopos est rendu par «cler perse », kianos n'est pas traduit, et inopos est déformé en sinopos. Influence de « sinopre » ou « sinople », du latin sinopis, lui-même issu du grec, et attesté à la fin du XII ${ }^{\mathrm{e}}$ siècle dans le Tristan avec le sens de « rouge ${ }^{57}$ ?

Les comparants renvoyant souvent à des réalités concrètes et peu universelles, et le conflit entre subjectivité et objectivité expliquent donc sans doute les résistances rencontrées par les traducteurs d'écrits sur les urines, tentés de laisser certains termes tels quels dès la mise en latin du Peri ouron de Théophile. Et sur cette vaste question, nous laisserons le dernier mot à Michel Pastoureau : « Nous tombons le plus souvent sur une phrase du type : Rouge (adjectif) : qui est de la couleur du sang, qui est de la couleur du feu. [...] Mais que dire d'autre, à part nommer des objets, des éléments naturels ou des êtres vivants de cette couleur $?^{58} »$.

\section{Difficultés, impossibilité, inventivité}

Abordons, pour terminer, à un autre réservoir à problèmes, à savoir la vaste production de traités d'astrologie en français qui prend son essor au XIII ${ }^{\mathrm{e}}$ siècle.

55. Ms. London, BL, Sloane 3525.

56. « orine pale, sozpale, bloie, lactine, blanche », cité par P. MEYER, « Manuscrits médicaux en français », Romania, 44 (1915-1917), p. 161-214 (p. 197).

57. A. J. GreImAs, Dictionnaire de l'ancien français jusqu'au milieu du XIv siècle, Paris, 1980 , p. 598.

58. M. Pastoureau, Une couleur ne vient jamais seule. Journal chromatique 2012-2016, Paris, 2017, p. 172. 
L'écrasante majorité des traductions se fonde sur des versions arabolatines, notamment celles réalisées par ou dans le sillage de Gérard de Crémone dans la Tolède du XII ${ }^{\mathrm{e}}$ siècle. On sait qu'avec Gérard de Crémone et ses associés, l'arabe était rendu littéralement, au prix de translittérations (ainsi, dans le domaine médical, soda pour sudâ’, « céphalée », ou alsechati pour as-sakta, « apoplexie »), et la traduction était pour ainsi dire un calque de l'original - ce qui explique que les unes comme les autres suscitèrent parfois des tentatives de révision. Mais il est des termes arabes qui résistent à tout essai d'adaptation et qui se fraieront dans le latin médiéval une place sous leur forme d'origine, malgré des déformations liées à de toujours possibles cacographies : un exemple parmi d'autres en est l'almubtez des astrologues arabes, à savoir la planète dominante qu'il s'agit de trouver pour un jugement astrologique assuré, qu'on trouve sous la forme almubtaz dans les premières traductions latines d'Alcabitius et Albumasar. Si l'on excepte une oscillation vocalique entre «e » et «a », liée à la variabilité des voyelles de la langue sémitique d'origine, un phénomène d'inversion entre « $\mathrm{u}$ » et « $\mathrm{m} »$, ou la disparition des sons « $\mathrm{b} »$ et $« \mathrm{z} »$ dans la forme almuten qu'il pourra revêtir ${ }^{59}$, le terme est intégré tel quel dans les textes latins, épargné par les flexions de la langue, puis dans les premiers textes d'astrologie en français, comme peut le montrer entre autres une rapide confrontation entre un extrait du De urina non visa achevé par Guillaume l'Anglais en 1220 et la traduction de la Compilatio de astrorum scientia de Léopold d'Autriche réalisée à la fin du XIII ${ }^{\mathrm{e}}$ siècle ${ }^{60}$. Le traducteur anonyme a renoncé à toute translation au profit d'une exportation pure et simple d'un mot d'origine arabe, comme on l'a vu plus haut avec les trois dénominations de couleurs prises au grec de Théophile, et comme ce sera le cas de beaucoup de termes spécifiques hérités de la science des astres arabe tels hyleg, alcocoden, azimene, etc.

De ce point de vue, un traducteur constitue l'exception : je veux parler d'Hagin le Juif, qui réalisa en 1273, pour Henri Bate de Malines, la version française de quatre traités astrologiques composés en langue hébraïque par Abraham ibn Ezra ; faute de place, on prendra des exemples dans le Commencement de Sapience, conservé dans un seul témoin du XIII ${ }^{\mathrm{e}}$ siècle, le manuscrit Paris, BnF, fr. 24276, f. 1r-66r (le manuscrit Paris,

59. Voir entre autres PtolÉmÉE, Le Livre unique de l'astrologie, trad. et comm. P. Charvet, Paris, 2000, Livre III, n. 210, p. 135 : « ce que la tradition latine des Arabes nomme almuten, c'est-à-dire la planète qui assume le rôle de planète dominante du thème de naissance ».

60. De urina non visa, chap. 7, éd. L. Moulinier-Brogi, Guillaume l'Anglais, le frondeur de l'uroscopie médiévale (XIII siècle), Édition commentée et traduction du De urina non visa, Paris/Genève, 2011, p. 158 : « Hic autem almubtaz domini urine corrumpens eius naturam suumque domicilium a se perspicatius perspectam causam manifestat. Locus almubtaz morbi fundamentum patefacit »; Li compilacion Leupol, ms. Paris, BnF, fr. 613, f. 56r : « Ichuis alumbtez manifeste le cause de le maladie et le cause et le nature et le siege et le singnourie. » 
Bnf, fr. 1351 est du $\mathrm{Xv}^{\mathrm{e}}$ siècle), et somme toute peu étudié depuis les recherches pionnières de Raphael Levy ${ }^{61}$.

Dans cette traduction, Hagin aussi semble renoncer parfois à toute traduction pour insérer dans son texte, tels quels, des mots figurant dans la version d'origine, ainsi kima, de l'hébreu kimah, המיכ, un vieux mot ouestsémitique que l'on trouve dans Job IX, 9 pour désigner l'amas d'étoiles des Pléiades ( « et ou signe du Buef sont 33 étoiles et Kima sur leur dos »), ou encore «Et celi qui est nés en un des degrés de Kima, qui est 13 degrés duques a 15 , il ara maladie en son oil ${ }^{62} »$.

Un terme comme creveice, pour désigner le signe du Cancer ( $\mathrm{La}$ creveice est des signes de l'iaue, femele, des signes de la nuit, et il est senestre, des signes de la terre, et il es trestornant car le tans se torne en $\left.\mathrm{li}^{63} \gg\right)$, pourrait pour sa part être une adaptation du yiddish krebs, « crabe », et on trouve aussi ici des mots importés tels quels de l'arabe : aldebaran, de l'arabe aldabaran, « la suivante » (une étoile de grande magnitude de la constellation du Taureau), ou encore alphardar, de l'arabe al-fardar, « domination ».

Hagin peut opter aussi pour des calques, comme chef $d u$ cheval, qui est sous sa plume une constellation septentrionale dont le nom vient de l'hébreu (« La 18 ${ }^{\mathrm{e}}$, c'est le chief du cheval, et ses estoiles sont $55^{64}$ »), et surtout geline, terme par lequel il désigne la constellation du Cygne. Cette appellation surprenante, et cette dégradation du cygne en poule, s'expliquent par le calque d'une appellation arabe, الددجاجةal-Da $\breve{g} \bar{a} \breve{g} a$, signifiant « la Poule », passé via le latin médiéval gallina, attesté pour la première fois avec ce sens dans la traduction de l'Almageste par Gérard de Crémone ${ }^{65}$. Le même phénomène expliquerait les expressions aigle cheant et aigle volant pour Lyra et Aquila.

Enfin et surtout, on doit à cet auteur de nombreuses créations linguistiques, comme ataisement, « situation d'une étoile ou d'une planète n'étant en aspect avec aucune autre » : «L'ataisement est que soit l'estoile en un signe et ne regarde a li nule estoile tant come le est iluec, ne il s'est departi de nule estoile ; et a la Lune avenra ainsinc pour la hativeté de

61. Voir R. Levy, The Astrological Works of Abraham ibn Ezra. A Literary and Linguistic Study with Special References to the old French Translation of Hagin, Baltimore, 1927 ; ID., Contribution à la lexicographie française selon d'anciens textes d'origine juive, Paris, 1932. Voir aussi P. PARIS, « Hagins le Juif, traducteur en français de plusieurs livres d'astronomie », Histoire littéraire de la France, 21 (1847), p. 499-503 ; et plus récemment F. FÉrY-HuE, « Hagin », dans G. HaSEnohr et M. Zink éd., Dictionnaire des lettres françaises. Le Moyen Âge, Paris, 1992, p. 659 ; J.-P. Boudet, dans C. Galderisi éd., Translations médiévales. Cinq siècles de traductions en français au Moyen Âge (XI ${ }^{e}-X V^{e}$ siècles). Étude et Répertoire, Turnhout, 2011, vol. 2.2, p. 1213-1217.

62. The Beginning of Wisdom : An Astrological Treatise by Abraham ibn Ezra, éd.

R. Levy et F. CANTERA, Baltimore, 1939, p. 42.

63. Ibid., p. 47.

64. Ibid., p. 34.

65. Liber Almagesti, ms. Paris, BnF, lat. 14738 (fin XII siècle), f. 118v. 
son aler $^{66}$. » Prinçoiement, pour sa part, signifie la dignité d'une planète, la situation favorable des trois planètes supérieures et des trois planètes inférieures par rapport à la marche du Soleil : «Et le prinçoiement, ce sont les.3. estoiles souvereines orientaus du Soleil des l'eure qu'il acommence a estre veus a la veue de l'ueil [...]. Et les.3. estoiles basses, acommencera leur prinçoiement de le tens qu'ils sont veus en occident aprés le couchement du Soleil ${ }^{67}$. » Reçoivement est forgé et employé comme synonyme de reception ( «Et le reçoivement est quant se conjoint l'estoile ou en conjonction ou en regart ensamble estoile qui est sire de sa meson, ou sire de la meson de s'oneur, ou sire de la meson de sa triplicité, ou son termine, ou ses faces, et il reçoit cele estoile $\left.{ }^{68} »\right)$, et reflambissement, créé à partir du latin flambellum, « torche, flambeau », signifie « éclat»: «Le disieme ou reflambissement de la clarté des 7 planetes ${ }^{69}$. » On rangera dans la même catégorie des créations linguistiques de Hagin sorticement, comme synonyme de « sort » au sens divinatoire ${ }^{70}$, torchonnoiement, alias obliquité, pour désigner la planète à son périgée $^{71}$, ou taillement de la clarté pour exprimer l'abscision de lumière ${ }^{72}$ :

Et le taillement de la clarté est sur .3. voies. L'une est que est l'estoile legiere conjongnant ou plus pesant de lui et ambedeus en .1. sine, et soit estoile tierce ou signe le secont; et avant que se conjongne le legier premier ensamble le pesant, retornera estoile qui est ou signe secont retornant arrieres, et entrera ou signe que illuec est le pesant et se conjoindra ensamble lui ; et donc detaillera il sa clarté de l'estoile premiere.

Hagin n'est pas, tant s'en faut, le seul traducteur créateur de néologismes en français ${ }^{73}$ ! Mais soulignons pour finir avec Raphael Levy que le Commencement de Sapience est unique en ce qu'il est le seul texte judéo-français écrit en caractères romans, par opposition à un vaste corpus de textes ou gloses en français écrits en caractères hébreux ${ }^{74}$, ou plutôt dicté à un

66. The Beginning of Wisdom..., p. 102.

67. Ibid., p. 106.

68. Ibid., p. 105.

69. Ibid., p. 32.

70. Ibid., p. 91.

71. Ibid., p. 103 : « Le torchonnoiement est quant est l'estoile en une des cheans. »

72. Ibid., p. 104-105.

73. Entre autres exemples, voir la liste de néologismes issus des traductions d'Aristote par Nicole Oresme dans J.-P. Rioux, J.-F. Sirinelli éd., Histoire culturelle de la France, t. I, M. Sot, J.-P. Boudet, A. Guerreau-Jalabert éd., Le Moyen Âge, Paris, 1997, p. 282. Voir à ce sujet, entre autres, T. STÄDTLER, « Le traducteur, créateur de néologismes. Le cas de Nicole Oresme », dans O. Bertrand, H. Gerner, B. StrumpF éd., Lexiques scientifiques et techniques..., p. 47-61.

74. R. LEVy, « L'aspect linguistique de la littérature judéo-française », Cahiers de l'AIEF, 9 (1957), p. 271-277 (p. 275), qui donne d'autres exemples de créations linguistiques d'Hagin hors du seul domaine astronomico-astrologique. 
certain Robert de Montdidier dans la maison d'Henri Bate de Malines, comme le dit le colophon ${ }^{75}$. Et surtout que le Commencement de Sapience, seul texte «français juif » du XIII 'siècle, marque une date sans précédent dans l'entreprise de traduction de traités astrologiques qui va crescendo à partir de 1300 : en effet, alors que, au moins pour les plus anciens textes astronomiques, on partait du latin médiéval comme langue source, un latin médiéval défini par Claude Buridant comme « la langue d'une Ideengemeinschaft, faisant fonction d'une communauté organique de sujets parlants, la langue de culture et de communication de la communauté intellectuelle européenne, la respublica clericorum $^{76}$ », Hagin, en traduisant, le premier, les traités d'Abraham ibn Ezra d'hébreu en français, se distingue par son choix de la langue source, et ses versions judéo-françaises à quatre mains serviront de base à des traductions latines dues respectivement à l'astronome Henri Bate de Malines pour le Commencement de sapience en 1292, et à Arnoul de Quincampoix, médecin de Philippe le Bel versé en hébreu, pour le Livre des élections et Le Livre des questions ${ }^{77}$. Sans doute fallait-il non seulement posséder un peu d'hébreu, mais aussi goûter l'inventivité linguistique pour apprécier le travail d'Hagin, car Pietro d'Abano, autre traducteur d'Ibn Ezra en latin, et notamment du Commencement de Sapience dès 1293, s'éloignera des versions données par Hagin...

Ce bref parcours ne se voulait pas autre chose qu'un survol d'un échantillon l'autre, entre différents domaines relevant de la littérature scientifique ou technique du Moyen Âge. À son terme, et au-delà de la variété des textes, des milieux linguistiques et des aires culturelles considérés, la Fachliteratur s'impose comme le lieu où la traduction de certains termes peut faire figure de problème insoluble dès lors qu'une condition préalable, à savoir l'identification correcte et précise d'un objet ou d'un élément naturel, n'est pas remplie. La littérature spécialisée implique déjà en soi la création ou la mise au point d'un vocabulaire ad hoc, approprié ; dès lors qu'on entend transférer ce savoir dans une ou d'autres langues, il faut trouver ou forger un lexique compréhensible, recevable dans la langue cible,

75. «Ci defines le Livre du commencement de sapience que fist Abraham even Azre, qui est interprété "maistre de aide", que translata Hagins li Juis de ebrieu en romans, et Obers de Mondidier escrivoit le roman, et fu fai a Malines en la meson sire Henri Bate, et fu fines en l'an de grace 1273, le lendemein de la Saint Thomas l'apostre » (cité par J.-P. Boudet dans C. GaLDERISI éd., Translations médiévales..., vol. 2.2, p. 1217).

76. Cité par S. DöRR, « La création du lexique astronomique et astrologique en ancien français. Observations méthodologiques et pratiques », dans O. BerTrand, H. Gerner, B. StrumpF éd., Lexiques scientifiques et techniques..., p. 176.

77. Voir à ce sujet J.-P. Boudet dans C. Galderisi éd., Translations médiévales..., vol. 2.2, p. 1213-1217; ID., Entre science et nigromance. Astrologie, divination et magie dans l'Occident médiéval (XII'-XV' siècle), Paris, 2006, notamment p. 56 et 165. 
et l'emprunt s'avère une possibilité largement exploitée par les traducteurs, ici de l'allemand comme du grec, de l'hébreu ou de l'arabe, et vers le latin ou le français. Encore faut-il rappeler que, selon les lieux, les langues source et cible peuvent être apparentées ou non et que l'assimilation des emprunts en revêtira des visages divers. Mais le maintien pur et simple d'un terme venu d'ailleurs n'est qu'une des voies dans lesquelles se sont engagés les traducteurs de textes spécialisés, et on a vu plus haut des exemples de recours à l'adaptation, à la glose ou aux binômes de synonymes ${ }^{78}$, sans que ces emplois aient rien de systématique au sein d'une même translation. Soulignons enfin que la traduction était et est toujours un art, et que nos sondages nous ont fait rencontrer une gamme allant du renoncement à la création linguistique, magnifiquement illustrée par Hagin.

Laurence Moulinier-Brogi - Université Lumière-Lyon 2, UMR 5648 (CIHAM)

\section{Y a-t-il de l'intraduisible ? Quelques exemples de résistances dans les écrits scientifiques du Moyen Âge}

L'histoire des traductions en vulgaire de la littérature technique ou scientifique, appelée Fachliteratur en allemand, est riche en problèmes de terminologie, et souvent aussi de philologie, des erreurs de copie parfois perpétuées par des éditions ayant pu offrir des obstacles durables à l'identification correcte de tel ou tel objet. L'article donne un rapide panorama des difficultés inhérentes aux translations d'écrits scientifiques ou techniques en s'intéressant aux écueils liés à la traduction de noms d'espèces locales, notamment végétales, par la transmission fidèle des noms de couleurs d'une aire linguistique à une autre, ou encore par l'adoption de nouveaux termes introduits par les traductions arabo-latines en matière de science des astres à partir du $\mathrm{XII}$ e siècle. Ces sondages dans différents champs de la science médiévale révèlent des résistances en matière d'identification, de transposition du particulier à l'universel, ou d'intégration dans une langue cible. II rappelle au passage combien le gisement textuel constitué par les glossaires, les listes de synonymes ou autres lexiques, encore à défricher malgré de récentes avancées, a pu être d'un précieux secours pour les lecteurs du Moyen Âge, et peut l'être encore pour les modernes.

écrits scientifiques, Fachliteratur, Hagin le Juif, Hildegarde de Bingen, Liber subtilitatum, noms de couleurs, noms de plantes, science des astres, traduction, uroscopie, vernacularisation

\section{Are some things untranslatable ? Certain cases of resistance in the scientific writings of the Middle Ages}

The history of the vernacular translations of the scientific or technical literature of the Middle Ages, the so-called Fachliteratur in German, presents many terminological and philological problems : these texts sometimes contain copy errors carried over into subsequent editions, and misreading is often the primary reason why specific objects are incorrectly identified.

78. Voir notamment C. Buridant, « Les binômes synonymiques. Esquisse d'une histoire des couples de synonymes du Moyen Âge au XVII siècle », Bulletin du Centre d'analyse du discours de l'Université de Lille III, Synonymies, 4 (1980), p. 5-76. 
This article aims to provide a sample of the difficulties encountered in the translation of scientific or technical writing. It considers the translation of names of different species, in particular botanical ones, which are often very locally specific and sometimes even extinct. It also looks at the issue of accurately transmitting the names of colors from one linguistic area to another, and, lastly, the adoption of new terms introduced from the twelfth century onward into the medieval Western world by the Latin translations from Arabic about the science of the stars. This brief overview of various different fields of medieval science reveals that there was some resistance when it came to identifying and transposing the peculiar to the universal, or integrating new words into a target language. It also briefly touches upon the significance of medieval glossaries, lists of synonyms, and lexicons, which were very useful for medieval readers and can still be of great help for modern readers or translators.

color names, Fachliteratur, Hagin the Jew, Hildegard of Bingen, Liber subtilitatum, names of plants, science of celestial bodies, scientific writing, translation, uroscopy, vernacularization 